\title{
Effects of 6 Weeks Classification-guided Exercise on Pain and Functionality in Subgroups of Nonspecific Chronic Low Back Pain
}

\author{
Kyung-hee Noh, PT, Ph.D. ${ }^{1}$; Jae-seop Oh, PT, Ph.D. ${ }^{2}$ \\ ${ }^{1}$ Noh Kyunghee Movement Training Center, Pusan, South Korea \\ ${ }^{2}$ Department of Physical Therapy, Inje University, Gimhae, South Korea
}

Background A physical therapeutic diagnosis, classified on the basis of underlying mechanics that produce disabilities, will help to predict the disorders and will be a guideline for determining the appropriate intervention. Fewer studies have investigated specific interventions via movement system impairment (MSI) classification of patients with nonspecific chronic low back pain (NCLBP).

Purpose The aim of the present study is to compare the effects of general exercises and specific exercises of classified subgroups in patients with NCLBP.

Study design Randomized Controlled Trial.

Methods A total of 34 patients with NCLBP participated, 17 in the specific exercise group by MSI classification (the MSI group) and 17 in the general exercise group (the general group). Before and after a 6-week intervention program, low back pain was assessed using the visual analog scale (VAS), and functional evaluation was assessed using the Sorensen test and functional movement screening test (FMS).

Results A significant time-by-group interaction was observed for the VAS, Sorensen, and FMS $(p<0.05)$. A post hoc paired $t$-test showed that the VAS and the Sorensen test scores decreased, and FMS increased significantly post-intervention in the MSI group $(p<0.01)$. The mean difference in the VAS, Sorensen, and FMS were significantly greater in the MSI group than in the general group $(p<0.05)$.

Conclusions The findings of this study suggest useful information for applying specific exercises in the subgroups, as determined by MSI classification.

Key words Functional movement screening test; Movement system impairment; Nonspecific chronic low back pain.

\section{INTRODUCTION}

The European guidelines recommend supervised exercise therapy as a first-line treatment in the management of nonspecific chronic low back pain (NCLBP). ${ }^{1}$ Exercise therapy is effective at improving function and decreasing pain in adults with NCLBP. ${ }^{2}$ In a systemic review, Middelkoop et al. found 37 studies on the topic of exercise therapy. ${ }^{3}$ Exercise therapy improved post-treatment pain intensity, disability, and long-term function. There is still no consensus
JMST

2019; 3(1): 7-13

Published Online Jun 30, 2019

pISSN 2635-8573 eISSN 2635-8581

Article History

Received 04 April 2019

Revised 04 April 2019

(1st)

Revised 10 May 2019

(2nd)

Accepted 10 May 2019

\section{CONTACT}

ysrehab@inje.ac.kr Jae-seop Oh,

Department of Physical

Therapy, Inje University,

Gimhae, South Korea

This is an Open-Access article distributed under the terms of the Creative Commons Attribution Non-Commercial License (http://creativecommons. cense (http://creativecommons.
org/licenses/by-nc/4.0) which org/licenses/by-nc/4.0) which permits unrestricted non-commercial use, distribution, and reproduction in any mework is properly cited. as to what kind of exercise should be used. ${ }^{1}$ In the European guidelines, recommendations are not given on the specificexercise to be set about populations with NCLBP. ${ }^{2}$ Hayden et al. reported no evidence to support the effectiveness of specific-exercise over others in patients with NCLBP. The lack of consistent evidence to support exercise interventions may be due to the heterogeneous study populations for comparison. ${ }^{4,5}$

Over several years, the custom has been to divide people with NCLBP into homogeneous populations of similar 
characteristics, in an effort to improve patient outcomes. The Agency for Healthy Care Policy and Research (AHCPR) suggested that optimal clinical management for lower-back problems depends on accurate diagnosis. ${ }^{6}$ There is growing evidence suggesting that patients with lower back pain can be subclassified into meaningful treatment groups. Homogeneous subgroups may result in more efficient treatment strategies and ountcomes ${ }^{7}$ and may increase the likelihood of responding to specific treatment. ${ }^{8}$

Movement system impairment (MSI) subgroups consist of five categories based on the testing of muscular stability, alignment, asymmetry, and flexibility of the lumbar spine, pelvis, and hip. The intervention purpose of subgroups is to correct factors predisposing or contributing to movement pattern impairments, alleviating stress on painful tissues, and allowing inflammation to subside. Spinal dysfunction is prevented and relieved through the optimal alignment and movement of the lumbopelvic region by the trunk muscles. ${ }^{9}$

Previously, there was intervention study for subjects in rotation with flexion syndrome into MSI subgroups. ${ }^{3}$ Marcie et al. reported on an intervention study for the rotation with extension syndrome group. ${ }^{10}$ Previous studies provided information about specific exercise via MSI classification, but case report. In Park et al.'s study, they determined the effects of the abdominal drawing-in maneuver on pelvic motions during active prone knee flexion in RE subgroup. ${ }^{11}$ Noh et al. suggested restricting pelvic rotation by balancing muscle activity during active straight leg raises in women with NCLBP. ${ }^{12}$

However, fewer studies have investigated specific interventions via MSI classification of patients with NCLBP. Particularly, no study has compared improving pain and functions between specific interventions via MSI classification and general exercises. Therefore, the aim of the present study was to compare the effects specific exercise in the subgroups via MSI classification and general exercises in patients with NCLBP.

\section{METHODS}

\section{Participants}

This study involved 34 patients with NCLBP who visited Hospital, in Pusan, Korea. Inclusion criteria included an onset of LBP of more than 12 weeks, an oswestry disability index (ODI) $>20$ points, and a visual analogue scale (VAS) score $>2$ points. Subjects were randomly grouped into a specific exercise group $(n=17)$ by their MSI classification or a general exercise group $(n=17)$. The MSI group was classified into five subgroups by MIS classification system.

Exclusion criteria were a history of spinal surgery in the previous three months, severe kyphosis, scoliosis or spinal stenosis, scheduled for surgery, history of more than one surgical procedure on the spine, cancer, rheumatoid arthritis, ankylosing spondylitis, neurological disease other than nerve root involvement at the spinal level, and inability to stand and walk without the use of walking aids. ${ }^{13}$ All participants reviewed and signed consent forms before volunteering, and the Institutional Research Review Committee of Inje University gave ethics approval.

\section{Outcome measures}

We measured before and after the six-week intervention program. To obtain reliable and valid scores of pain, psychosocial problems, disability, and physical function characteristics, we used the visual analog scale (VAS), fear avoidance belief questionnaire (FABQ), ${ }^{14}$ Oswestry disability index (ODI), patient-specific functional scale (PSFS), ${ }^{15}$ finger tip-to-floor test (FF), ${ }^{16}$ sorensen test, ${ }^{17}$ and functional movement screening test (FMS). ${ }^{18}$

Pain levels were evaluated using visual analog scale (VAS; $100 \mathrm{~mm}$ ). A 0 indicated no pain and $100 \mathrm{~mm}$ indicated the worst pain level. For the assessment of psychosocial problems, we used the Korean version of the FABQ (in physical activity and work). ${ }^{14}$ All participants were asked to complete the study questionnaires at the start and the end of the six-week study period. The ODI and PSFS were used to assess the level of disability associated with the NCLBP. The ODI has a percentage of total score ranging from 0 (minimal disability) to 50 (maximum disability), obtained by computing the sum of all responses. Physical functions used the FF, trunk extensor endurance test to evaluate back extensor endurance, and functional movement screening test as an integrated assessment tool .

\section{Intervention}

The general and MSI groups performed stretching for 10 to 15 minutes prior to the specified exercises. Both groups visited the hospital exercise center twice a week for six weeks and performed exercises for about 50 minutes. All subjects were instructed in three exercises per stage and holding 10 seconds 10 times for three sets per exercise. The general group applied methods suggested by McGill: pelvic tilting from a lying position, abdominal muscle strengthening exercises, back extensor strengthening exercises, oblique strengthening exercises, and Swiss ball coordination exercises (Figure 1). ${ }^{19,20}$ The MSI group consisted of strengthening exercises and corrective exercises: abdominal muscles, back extensor, gluteus maximus, and gluteus medius strengthening exercises (Figure 2); standing against 

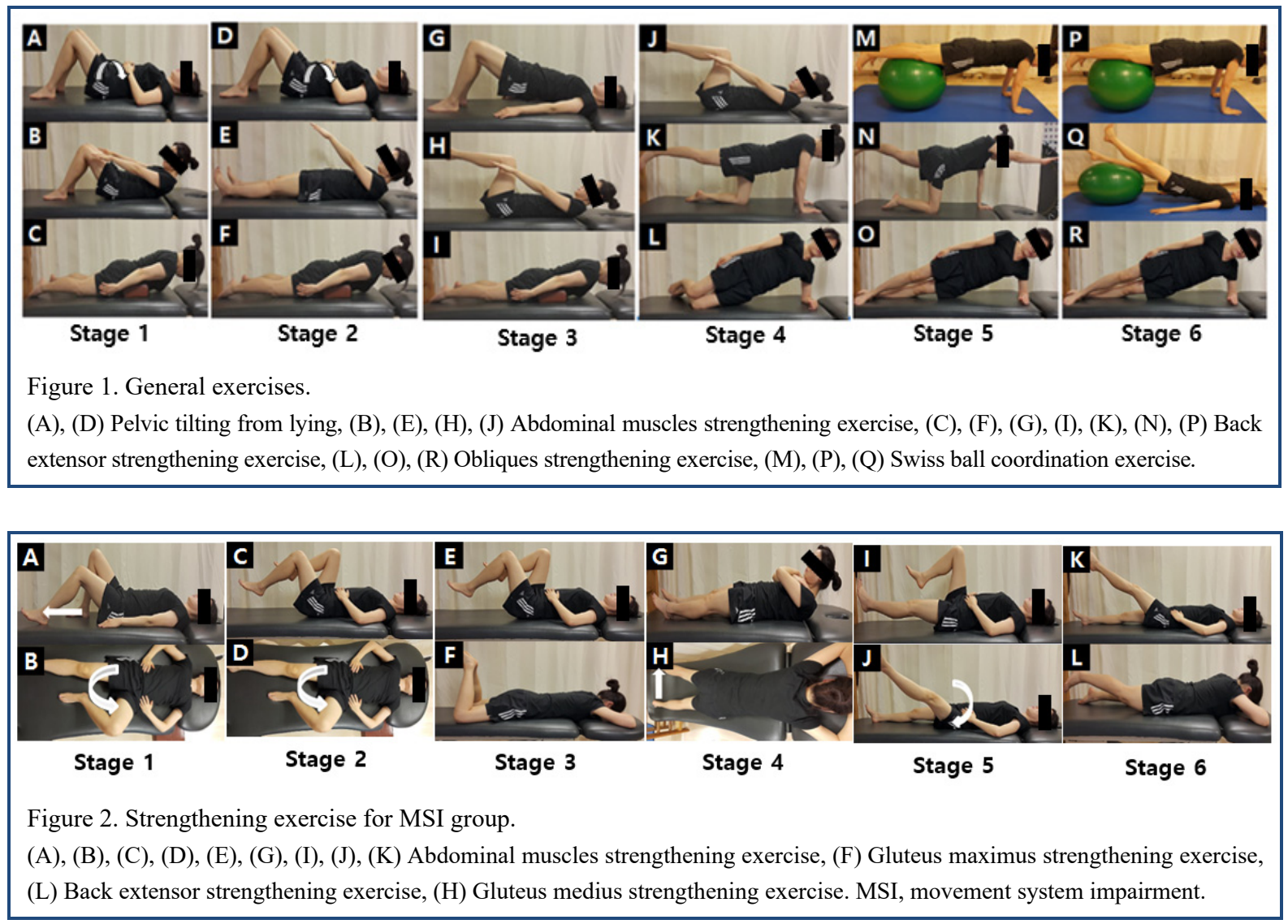

a wall, lateral spinal flexion, forward bending; spinal and hip flexion and forward bending; hip flexion with flat lumbar spine, knee extensions, and dorsiflexion; and rocking backward, latissimus dorsi muscle stretches, and hip rotations (Figure 3).

\section{Statistical analysis}

We measured the mean and standard deviation (SD) of the VAS, FABQ1,2, PSPF, ODI, FF and FMS scores. A twoway analysis of variance (ANOVA) was used to determine the main effect and any interaction between the VAS, FABQ1,2, PSPF, ODI, FF and FMS scores. The within- group factor was time (pre-intervention vs. post-intervention) and the between-group factor was group (MSI vs. general exercise). The significances of differences in demographic between subjects in the MSI and general exercise groups were analyzed by independent $t$-tests. Statistical Package for the Social Sciences (SPSS) ver. 18.0 (SPSS Inc., Chicago, USA) was used for analyses, and the significance was the adopted value of $p<0.05$.

\section{RESULTS}

The demographic and outcome measures data at baseline

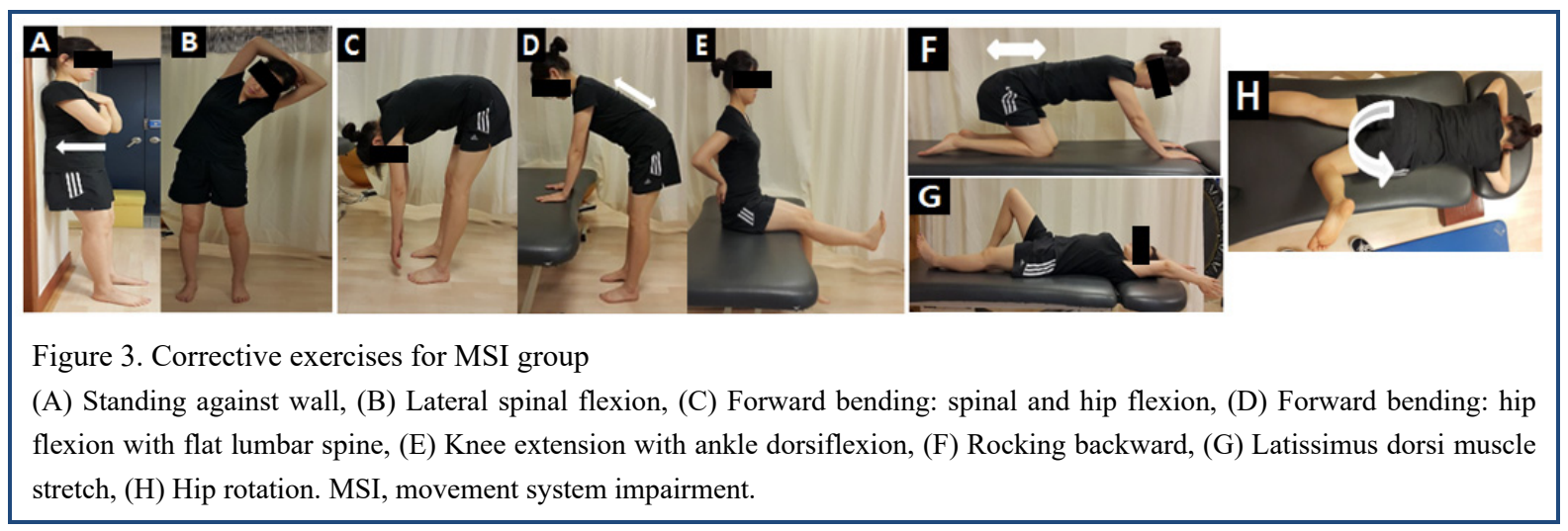


did not differ significantly between the two groups (Table 1). A significant time-by-group interaction was observed for the VAS, Sorenson Test, and FMS. An independent sample $t$-test demonstrated that the MSI exercise group had greater VAS (Table 2; $F=8.39, p<0.001$, mean difference $=21.81$ $\mathrm{mm}$ ), Sorenson test (Table 3; $F=8.39, p=0.014$, mean difference $=34.1 \mathrm{sec}$.), and FMS (Table $3 ; F=9.96, p=0.003$, mean difference $=2.7$ point) values. In addition, the post-hoc paired $t$-test showed that the Sorenson Test (Table 3; $p<0.001$ ) and FMS (Table $3 ; p<0.001$ ) significantly increased post-intervention versus pre-intervention in the MSI exercise group. Also, VAS (Table 2; $p<0.001$ ) significantly decreased post-intervention versus pre-

Table 1. Demographic characteristics of the subjects

$(n=34)$

\begin{tabular}{cccc}
\hline $\begin{array}{c}\text { Variable } \\
\text { (unit) }\end{array}$ & $\begin{array}{c}\text { General group } \\
(n=17)\end{array}$ & $\begin{array}{c}\text { MSI group } \\
(n=17)\end{array}$ & $p$ \\
\hline Age (years) & $42.12 \pm 13.20$ & $45.00 \pm 12.93$ & 0.525 \\
$\begin{array}{c}\text { Body mass } \\
\text { index }\left(\mathrm{kg} / \mathrm{m}^{2}\right)\end{array}$ & $23.42 \pm 2.87$ & $22.98 \pm 2.64$ & 0.654 \\
\hline
\end{tabular}

Data are expressed as mean $\pm \mathrm{SD}$.

${ }^{*} p<0.05$.

MSI, movement system impairment. intervention in the MSI exercise group.

A significant main effect was found for time for pain. VAS significantly decreased in the post-intervention versus pre-intervention in the general exercise group (Table 2; $40.01 \pm 2.09 \mathrm{~mm}$ vs. $48.82 \pm 22.05 \mathrm{~mm}, p=0.007$ ).

A significant main effect was found for time in the psychosocial problem. FABQ in physical activity significantly decreased in the post-intervention versus preintervention in the MSI exercise group (Table 4; $15.53 \pm 6.28$ vs. $20.76 \pm 6.54, p=0.012$ ). FABQ in work significantly decreased in the post-intervention versus pre-intervention in both groups (Table 4; general: $28.24 \pm 12.54$ vs. $37.35 \pm 12.86$, $p=0.002$; MSI: $26.76 \pm 13.38$ vs. $35.59 \pm 11.05, p<0.001$ ). There was no significant difference in psychosocial problems between the groups.

A significant main effect was found for time in disability. PSPF significantly increased in the post-intervention versus pre-intervention in the MSI exercise group (Table 5; $7.76 \pm 1.15$ vs. $5.58 \pm 1.47, p<0.001)$. ODI significantly decreased in the post-intervention versus pre-intervention in both groups (Table 5; general: $19.29 \pm 4.47$ vs. 27.18 \pm 6.64 , $p<0.001$; MSI: $17.53 \pm 7.13$ vs. $27.29 \pm 7.55, p<0.001$ ). However, there was no significant difference in disability between the groups.

Table 2. Changes in intensity of low back pain

$(n=34)$

\begin{tabular}{ccccccc}
\hline \multirow{2}{*}{ Variable } & \multirow{2}{*}{ Group } & Pre-intervention & Post-intervention & & \multicolumn{2}{c}{$p$} \\
\cline { 3 - 4 } \cline { 6 - 7 } & & Mean \pm SD & Mean \pm SD & & Within group & Between groups \\
\hline \multirow{2}{*}{ VAS (mm) } & General & $48.82 \pm 22.05$ & $40.01 \pm 20.92$ & & $0.007^{*}$ & $0.007^{*}$ \\
& MSI & $49.41 \pm 21.64$ & $27.65 \pm 16.78$ & & $<0.001^{*}$ & \\
\hline
\end{tabular}

Data are expressed as mean $\pm \mathrm{SD}$.

${ }^{*} p<0.05$.

MSI, movement system impairment; VAS, visual analog scale.

Table 3. Change in outcome measure of physical function between pre- and post-interventions

$(n=34)$

\begin{tabular}{|c|c|c|c|c|c|}
\hline \multirow{2}{*}{ Vriables } & \multirow{2}{*}{ Group } & Pre-intervention & Post-intervention & \multicolumn{2}{|c|}{$p$} \\
\hline & & Mean \pm SD & Mean \pm SD & Within group & Between groups \\
\hline \multirow{2}{*}{$\mathrm{FF}(\mathrm{cm})$} & General & $4.76 \pm 10.90$ & $8.44 \pm 7.50$ & 0.068 & \multirow{2}{*}{$0.026^{*}$} \\
\hline & MSI & $3.29 \pm 7.79$ & $1.71 \pm 11.13$ & 0.225 & \\
\hline \multirow{2}{*}{ Sorenson test (sec.) } & General & $33.47 \pm 20.49$ & $46.53 \pm 23.03$ & $0.019^{*}$ & \multirow{2}{*}{$0.014^{*}$} \\
\hline & MSI & $41.29 \pm 29.22$ & $76.71 \pm 42.38$ & $<0.001^{*}$ & \\
\hline \multirow{2}{*}{ FMS (point) } & General & $10.29 \pm 1.61$ & $11.82 \pm 1.38$ & $<0.001^{*}$ & \multirow{2}{*}{$0.003^{*}$} \\
\hline & MSI & $11.18 \pm 1.70$ & $13.82 \pm 1.47$ & $<0.001^{*}$ & \\
\hline
\end{tabular}

Data are expressed as mean $\pm \mathrm{SD}$.

$* p<0.05$.

FF, finger tip-to-floor; FMS, functional movement screening, MSI, movement system impairment. 
Table 4. Changes in outcome measure of psychosocial problem

$(n=34)$

\begin{tabular}{|c|c|c|c|c|c|}
\hline \multirow{2}{*}{ Variable } & \multirow{2}{*}{ Group } & Pre-intervention & Post-intervention & \multicolumn{2}{|c|}{$p$} \\
\hline & & Mean \pm SD & Mean \pm SD & Within group & Between groups \\
\hline \multirow{2}{*}{$\begin{array}{c}\text { FABQ in physical } \\
\text { activity }\end{array}$} & General & $21.06 \pm 6.74$ & $19.29 \pm 12.21$ & 0.449 & \multirow{2}{*}{0.245} \\
\hline & MSI & $20.76 \pm 6.54$ & $15.53 \pm 6.28$ & $0.012^{*}$ & \\
\hline \multirow{2}{*}{ FABQ in work } & General & $37.35 \pm 12.86$ & $28.24 \pm 12.54$ & $0.002^{*}$ & \multirow{2}{*}{0.927} \\
\hline & MSI & $35.59 \pm 11.05$ & $26.76 \pm 13.38$ & $<0.001^{*}$ & \\
\hline
\end{tabular}

Data are expressed as mean $\pm \mathrm{SD}$.

${ }^{*} p<0.05$.

FABQ, fear-avoidance beliefs questionnaire; MSI, movement system impairment.

Table 5. Change in outcome measure of disability between pre-and post- interventions

$(n=34)$

\begin{tabular}{clcccccc}
\hline \multirow{2}{*}{ Variable } & \multirow{2}{*}{ Group } & Pre-intervention & Post-intervention & & \multicolumn{2}{c}{$p$} \\
\cline { 3 - 4 } \cline { 6 - 7 } & & Mean \pm SD & Mean \pm SD & & Within group & Between groups \\
\hline \multirow{2}{*}{ PSPF } & General $(n=17)$ & $4.94 \pm 1.75$ & $6.12 \pm 1.90$ & & 0.058 & .257 \\
& MSI $(n=17)$ & $5.58 \pm 1.47$ & $7.76 \pm 1.15$ & & $<0.001^{*}$ & \\
\multirow{2}{*}{ ODI } & General $(n=17)$ & $27.18 \pm 6.64$ & $19.29 \pm 4.47$ & & $<0.001^{*}$ & .338 \\
& MSI $(n=17)$ & $27.29 \pm 7.55$ & & $17.53 \pm 7.13$ & & $<0.001^{*}$ & \\
\hline
\end{tabular}

Data are expressed as mean \pm SD.

${ }^{*} p<0.05$.

MSI, movement system impairment; PSPF, patient-specific functional scale; ODI, oswestry disability index.

\section{DISCUSSION}

We compared the effect of specific exercise by MSI classification and general exercise in patients with NCLBP. Our results show that the MSI exercise and general exercise groups decreased pain, psychosocial problems, and disability, and increased physical function in patients with NCLBP. However, reduced FABQ in physical performance and PSPF were only found in the specific exercise by MSI classification.

In this study, pain significantly decreased in both groups, but the MSI group showed a larger mean difference. Pain could be distributed on unstable segments of lumbar spine in radiological confirmation, ${ }^{16}$ and could be induced due to muscle imbalance ${ }^{19,21}$ exaggerating the flexibility of the site. ${ }^{9}$ Also, pain related to balanced isometric contraction and movement control provided by the trunk muscles. ${ }^{9}$ The general exercise group performed only strength training of the abdominal and paraspinal muscles. ${ }^{22}$ Previous studies reported significantly decreased pain in the general exercise-only group after the end of an eight-week exercise period; it is the same with our findings. However, the pain improved in the specific exercise group by MSI classification, likely due to restricted excessive movement of the flexible lumbar segment, as well as strengthening during the exercise. ${ }^{9}$ For the MSI group, exercise may be appropriate for muscle balance and controlling excessive movement.

Our findings showed improved physical function in both groups. FMS is positively related to core strength. ${ }^{23}$ Tasks of FMS need individual abilities, requiring a combination in physical activity. Five tasks in FMS require trunk stability. Also, back endurance plays a very important role in spinal stability during prolonged physical activity. MSI and general exercise may provide trunk stability. However, the MSI group showed a larger difference in the mean difference. Noh et al. ${ }^{11}$ and Park et al. ${ }^{12}$ suggested that minimizing the method of unwanted lumbopelvic rotation increased the activation of abdominal muscles better than conventional methods while maintaining active straight leg raising. These results in the MSI group would be due to restricted excessive lumbopelvic motion by isometric trunk muscles during movement of the upper or/and lower extremities. Pre-intervention FMS scores of our subjects with NCLBP and pre-intervention FMS scores were approximately 10 points. If football players' scores were 14 or less for FMS performance, ${ }^{24}$ this predicted their injury. After the six-week intervention in the MSI group, FMS scores averaged 13.83. Although the MSI group's postintervention score was less than 14 , this result is clinically 
meaningful because of subjects with NCLBP in our study.

The present study found that the self-report questionnaires for psychosocial problems and disability improved in both groups, but there was no significant difference between the groups. In the systemic reviews, exercise therapy slightly reduced sick leave during the first year and decreased the proportion of patients who had not returned to work at 1 year. ${ }^{19}$ Brox et al. reported that cognitive intervention and exercise therapy were effective in the FABQ of patients with NCLBP. ${ }^{25}$ Our results showed that there were effective psychosocial problems and disability in both groups in NCLBP. A corrective exercise in MSI exercise is a method restraining excessive motion of the lumbar spine to a specific direction that elicits or increases symptoms. Such exercise was suggested to reduce the fear of physical activities in the subgroup by MSI classification.

This study had some limitations. First, our study had a six-week exercise intervention, and we immediately measured the outcome after the intervention. NCLBP has a high recurrence rate. Future studies should investigate a longer exercise period and a long-term follow-up. Thus, these results cannot be generalized to patients with NCLBP because the sample size was small.

\section{CONCLUSIONS}

The study compared the effects of specific exercise in the subgroups by classification and general exercises in patients with NCLBP. Our findings showed that the VAS score decreased and the Sorensen test and FMS increased postintervention within the MSI group. The mean difference in the VAS, the Sorensen test, and FMS scores were significantly greater in the MSI group than in the general group. Therefore, a specific intervention based on MSI, applied to a homogenous group of NCLBP patients, may provide appropriate therapeutic exercises in the clinical setting.

\section{Key Points}

Question Does applying specific exercises by MSI classification improve pain and function compared to general exercises?

Findings There was statistically significant improvement on pain and physical function in the MSI group than in the general group.

Meaning This study may suggest useful information for applying specific exercises in the subgroups, as determined by MSI classification.

\section{Article information}

Conflict of Interest Disclosures: None.

Funding/Support: None.

Acknowledgment: None.

Ethic Approval: IRB was approved at the Inje University Ethics Committee for Human Investigations.

\section{REFERENCES}

1. Airaksinen O, Brox JI, Cedraschi C, et al. Chapter 4. European guidelines for the management of chronic nonspecific low back pain. Eur Spine J. 2006;15(2): S192-S300.

2. Hayden J, Van Tulder MW, Malmivaara A, et al. Exercise therapy for treatment of non-specific low back pain (Review). The Cochrane Library. 2005;3:CD000335.

3. Van Dillen LR, Sahrmann SA, Wagner JM. Classification, intervention, and outcomes for a person with lumbar rotation with flexion syndrome. Phys Ther. 2005;85(4): 336-351.

4. Leboeuf-Yde C, Lauristen JM, Lauritzen T. Why has the search for causes of low back pain largely been nonconclusive? Spine. 1997;22(8):877-881.

5. Sorensen BF. Physical measurements as risk indicators for low-back trouble over a one-year period. Spine. 1984;9(2):106-119.

6. Kent P, Keating J. Do primary-care clinicians think that nonspecific low back pain is one condition? Spine. 2004;29(9):1022-1031.

7. Rose SJ. Musing on diagnosis. Phys Ther. 1988;68(11): 1665.

8. Abenhaim L, Rossignol M, Gobeille D, et al. The prognostic consequences in the making of the initial medical diagnosis of work-related back injuries. Spine. 1995;20(7):791-795.

9. Delitto A, Erhard RE, Bowling RW, et al. A treatment based classification approach to low back syndrome: identifying and staging patients for conservative treatment. Phys Ther. 1995;75(6):470-489.

10. Marcie HH, Van Dillen LR. The inter-tester reliability of physical therapists classifying low back pain problems based on the Movement System Impairment classification system. $P M \& R$. 2009; 1(2):117-126.

11. Park KN, Cynn HS, Kwon OY, et al. Effects of abdominal drawing- in maneuver on muscle activity, pelvic motions, and knee flexion during active prone knee flexion in patients with lumbar extension rotation syndrome. Arch Phys Med Rehabil. 2011;92(9):14771483.

12. Noh KH, Kim JW, Oh JS, et al. The influence of dual 
pressure biofeedback units on pelvic rotation and abdominal muscle activity during the active straight leg raise in women with chronic lower back pain. $J$ Phys Ther Sci. 2014;26(5):717-719.

13. Petersen T, Laslett M, Thorsen H, et al. Diagnostic classification of non-specific low back pain. A new system integrating patho-anatomic and clinical categories. Physiother Theor Pract. 2003;19(4): 213-237.

14. Waddell G. The back pain revolution, 2nd ed. London: Churchill-Livingstone; 2004.

15. Spratt KF, Lehmann TR, Weinstein JN, et al. A new approach to the low-back physical examination: behavioral assessment of mechanical signs. Spine. 1990;15(2): 96-102.

16. Perret C, Poiraudeau S, Fermanian J, et al. Validity, reliability, and responsiveness of the Fingertip-to-Floor Test. Arch Phys Med Rehabil. 2001;82(11):1566-1570.

17. Sorenson BF. Physical measurements as risk indicators for low-back trouble over a one-year period. Spine. 1984;9(2):106-119.

18. Ibrahim AI, Muaidi QI, Abdelsalam MS, et al. Association of postural balance and isometric muscle strength in early-and middle school age boys. J Manip Physiol Ther. 2013;36(9):633-643.

19. Arokoski JP, Kankaanpää M, Valta T, et al. Back and hip extensor muscle function during therapeutic exercises. Arch Phys Med Rehabil. 1999;80(7):842-850.
20. Koumantakis GA, Watson PJ, Oldham JA. Trunk muscle stabilization training plus general exercise versus general exercise only: randomized controlled trial of patients with recurrent low back pain. Phys Ther. 2005;85(3):209-225.

21. Kiesel K, Plisky P, Butler R. Functional movement test scores improve following a standardized off-season intervention program in professional football players. Scand J Med Sci Sports. 2011;21(2):287-292.

22. McGill SM, Childs A, Libenson C. Endurance times for low back pain stabilization exercise: clinical targets for testing and training from a normal database. Arch Phys Med Rehabil. 1999;80(8):941-944.

23. Mitchell UH, Johnson AW, Adamson B. Relationship between functional movement screen scores, core strength, posture, and BMI in school children in Moldova. J Strength Cond Res. 2015;29(5):1172-1179.

24. Cook G, Burton L, Hoogenboom B. Pre-participation screening: the use of fundamental movements as an assessment of function-part 1. N Am J Spots Phys Ther. 2006;1(2):62-72.

25. Brox JI, Sorenson R, Friis A, et al. Randomized clinical trial of lumbar instrumented fusion and cognitive intervention and exercises in patients with chronic low back pain and disc degeneration. Spine. 2003;28(17): 1913-1921. 